\title{
Ultrasonic Pretreatment of Wheat Straw in Oxidative and Nonoxidative Conditions Aided with Microwave Heating
}

\author{
Madeleine J. Bussemaker, ${ }^{* \dagger}$ Xindong $\mathrm{Mu}{ }^{\ddagger}$ and Dongke Zhang ${ }^{\dagger}$ \\ $\dagger$ Centre for Energy (M473), The University of Western Australia, 35 Stirling Highway, Crawley, WA 6009, Australia \\ ${ }^{\ddagger}$ Key Laboratory of Biobased Materials, Qingdao Institute of Bioenergy and Bioprocess Technology, Chinese Academy of Sciences, \\ 189 Songling Road, Laoshan District, Qingdao 266101, Shandong, China
}

\begin{abstract}
Ultrasound was shown to enhance pretreatment of lignocellulose for biofuel and biorefinery applications and can augment oxidative processes, yet few studies have combined ultrasound with an oxidative environment for the pretreatment of lignocellulose. In the present contribution, pretreatment of a wheat straw using a combination of ultrasound with two oxidative pretreatments, peracetic acid and hydrogen peroxide, as well as water and acetic acid was tested. The experimentation was conducted in an ultrasonic-microwave reactor with a comparison to silent pretreatment with microwave heating alone. In addition, thermal heating was compared to microwave heating for the water pretreatment. Ultrasound produced a higher purity solid residue for all chemical pretreatments. However, ultrasound pretreatment reduced the delignification efficacy by up to $50 \%$, attributed to lignin condensation. The chemical treatments were affected by sonolysis reactions and altered the recoverability of the solubilized carbohydrates.
\end{abstract}

\section{INTRODUCTION}

Pretreatment aims to separate or enhance accessibility to the three main components of lignocellulose, cellulose, lignin, and hemicellulose, and was the subject of several reviews. ${ }^{1-3}$ Ultrasound as a pretreatment option for lignocellulose was also reviewed, ${ }^{4}$ and it was revealed that ultrasound enhanced delignification, accessibility, and selectivity of treatment of lignocellulose. In addition, the literature review highlighted the gap in the literature of the combination of ultrasound with concurrent chemical pretreatment. Ultrasound is known to augment oxidative processes, ${ }^{5}$ and the degradation of phenol with hydrogen peroxide and peracetic acid was improved with ultrasound. ${ }^{6,7}$ Therefore, in this contribution, oxidative pretreatments combined with ultrasonic pretreatment were considered, compared to ultrasound combined with two nonoxidative pretreatments, water and acetic acid.

Peracetic acid is selective toward lignin which can augment pretreatment of a variety of biomass types. ${ }^{8-15}$ Delignification with peracetic acid occurs via hydroxonium ion $\left(\mathrm{HO}^{+}\right)$attack of lignin, which leads to oxidation reactions to break up the lignin macromolecule. ${ }^{3}$ Although augmentation of peracetic acid degradation of phenol with ultrasound was observed, ${ }^{6}$ no previous reports on the combination of ultrasound with peracetic acid for lignocellulosic pretreatment were found.

Hydrogen peroxide is often used in combination with alkaline as a pretreatment. ${ }^{16-18}$ The delignification of wheat straw with low concentration (ca. 1\%) hydrogen peroxide was promoted at $\mathrm{pH} 11.5$ due to the production of the $\mathrm{HOO}^{-}$ anion, which in turn promoted the production of hydroxyl $(\bullet \mathrm{OH})$ and superoxide $\left(\mathrm{O}_{2}^{-} \cdot\right)$ radicals. Similarly, the promotion of radicals with ultrasound and a Fenton reagent enhanced solubilized lignin degradation and improved enzymatic saccharification of kenaf powder. ${ }^{19}$ Hydrogen peroxide has alternate oxidative delignification mechanisms to peracetic acid, but a direct comparison of the two oxidative pretreatments in ultrasonic conditions has not been previously explored.

Acetic acid pretreatment aims to degrade hemicellulose and disrupt the lignin structure through solvation of lignin fragments. ${ }^{3}$ Furthermore, it was found ultrasound improved delignification by up to 13.8 and $9.9 \%$ and hemicellulose solubilization by up to 11.2 and $6.9 \%$ in water and $60 \%$ acetic acid, respectively, for the treatment of olive tree prunings. ${ }^{20}$

Microwave heating was utilized for pretreatment of lignocellulose $\mathrm{e}^{21-27}$ and for corn starch pretreatment for bioethanol production combined with ultrasound. ${ }^{28}$ In general, microwave heating reduced pretreatment time and provided an efficient heating mechanism. Microwave heating energizes polar bonds and creates heat energy throughout the substance and thus is more uniform and rapid than thermal heating.

Peracetic acid and hydrogen peroxide degrade lignin via alternative mechanisms, and acetic acid degrades hemicellulose. Ultrasound was hypothesized to augment the oxidative power of hydrogen peroxide and peracetic acid and to increase the efficacy of acetic acid pretreatment. A comparative study of ultrasonic pretreatment in these two oxidative conditions is novel. Moreover, acetic acid and water provide a comparison of nonoxidative pretreatment to the oxidative pretreatments in ultrasound. In addition, it was also hypothesized that microwave heating is a convenient alternative to thermal heating. The combination of ultrasound and microwave heating for the pretreatment of lignocellulose is novel. These hypotheses were tested using a model lignocellulose, wheat straw, treated with and without ultrasound for peracetic acid, hydrogen peroxide, and acetic acid solutions. Then, three

Received: April 14, 2013

Revised: July 11, 2013

Accepted: August 9, 2013

Published: August 9, 2013 
control pretreatments in water were conducted, using different heating mechanisms: microwave, microwave plus ultrasound, and thermal heating.

\section{EXPERIMENTAL SECTION}

2.1. Material. Wheat straw was sourced from the Wheatbelt region of Western Australia and was manually downsized prior to processing in a Waring Blender for $1 \mathrm{~min}$. The resultant particle size ranged up to $5 \mathrm{~mm}$ in diameter with a particle size distribution of $1.8 \%, 15.0 \%, 30.5 \%$, and $49.0 \%$ for ranges, $>2$ $\mathrm{mm}, 2-1 \mathrm{~mm}, 1-0.5 \mathrm{~mm}$, and $<0.5 \mathrm{~mm}$, respectively. The raw composition of the feedstock was determined via the analytical methods described below for the solid residue analysis.

2.2. Pretreatment Methods. In order to test the hypothesis that ultrasound could augment oxidative pretreatments, four chosen solvents were tested with and without ultrasound: approximately $20 \%(\mathrm{w} / \mathrm{w})$ peracetic acid, $1 \%(\mathrm{v} / \mathrm{v})$ hydrogen peroxide, $25 \%(\mathrm{v} / \mathrm{v})$ acetic acid, and water. Additional experiments were conducted using heating with an oil bath using water as the solvent. Hydrogen peroxide loading was chosen based on previous pretreatments of wheat straw in the literature. ${ }^{18}$ Peracetic acid concentration was chosen based on preliminary experiments of our laboratory. Peracetic acid pretreatment of wheat straw at concentrations 15,20 , and $25 \%$ (w/w) found that delignification benefited from a concentration increase of 15 to $20 \%$ but not from 20 to $25 \%$ (results not shown). The peracetic acid was made based on previous pretreatment schemes: ${ }^{29}$ Briefly, glacial acetic acid and 30\% hydrogen peroxide were mixed in the ratio of $3: 2(\mathrm{v} / \mathrm{v})$ with the addition of $\mathrm{H}_{2} \mathrm{SO}_{4}$ as a catalyst $(0.1 \% \mathrm{v} / \mathrm{v})$. An aqueous acetic acid pretreatment solution was utilized to provide a comparison to the oxidative pretreatment and to minimize variability in physicochemical properties which are known to influence ultrasonic effects. A nominal concentration of $25 \%$ was chosen based on a previous optimization study for the pretreatment of rice straw. ${ }^{30}$ All chemical reagents were sourced from Sinopharm chemical reagents, except for acetic acid which was sourced from FUYU chemicals.

Since the focus of the investigation was the combination of ultrasound with different pretreatments, the remaining parameters were kept constant. For all treatments, the loading of wheat straw was $1 / 20(\mathrm{~g} / \mathrm{mL})$, chosen based on previous work of our laboratory with wheat straw. The treatment volume of $400 \mathrm{~mL}$ was utilized based on visible observations of biomass mixing under ultrasound within the restrictions of the reactor vessel. The temperature was kept constant at $50 \pm 5{ }^{\circ} \mathrm{C}$ by ultrasonic energy input, microwave or manual monitoring; depending on the experimental conditions, this temperature was chosen in order to maximize sonochemical effects in aqueous conditions, as per a majority of previous ultrasonic pretreatments. ${ }^{4}$ The microwave and ultrasonic treatments were conducted using a XiangHu combined computer microwave instrument (XH 300A). Wheat straw was suspended in the treatment solution with stirring prior to treatments. For treatments with only microwave heating, the initial power of the microwave was set to $800 \mathrm{~W}$ and preheat time to $50{ }^{\circ} \mathrm{C}$ was $90 \mathrm{~s}$, after which the temperature was set at $50{ }^{\circ} \mathrm{C}$ for $30 \mathrm{~min}$, monitored automatically using power input of the microwave reactor. For ultrasonic pretreatments combined with microwave preheating, the same preheat conditions were used, and after preheating the microwave power input was turned off, while the ultrasonic input was turned on. The ultrasonic input was pulsed at a ratio of $1: 2$, on:off at frequency of $25 \mathrm{kHz}$. The input power of the ultrasound was set to $1600 \mathrm{~W}$ which corresponded to a dissipated power rating, determined calorimetrically of, $74.8 \pm$ $0.1 \mathrm{~W} \mathrm{~L}^{-1}$, following methods outlined by Koda et al. ${ }^{31}$ At these settings the temperature of the sonicated solution was not increased by more than $5{ }^{\circ} \mathrm{C}$ during pretreatment as shown in a typical heating curve in Figure 1 . The treatment time and
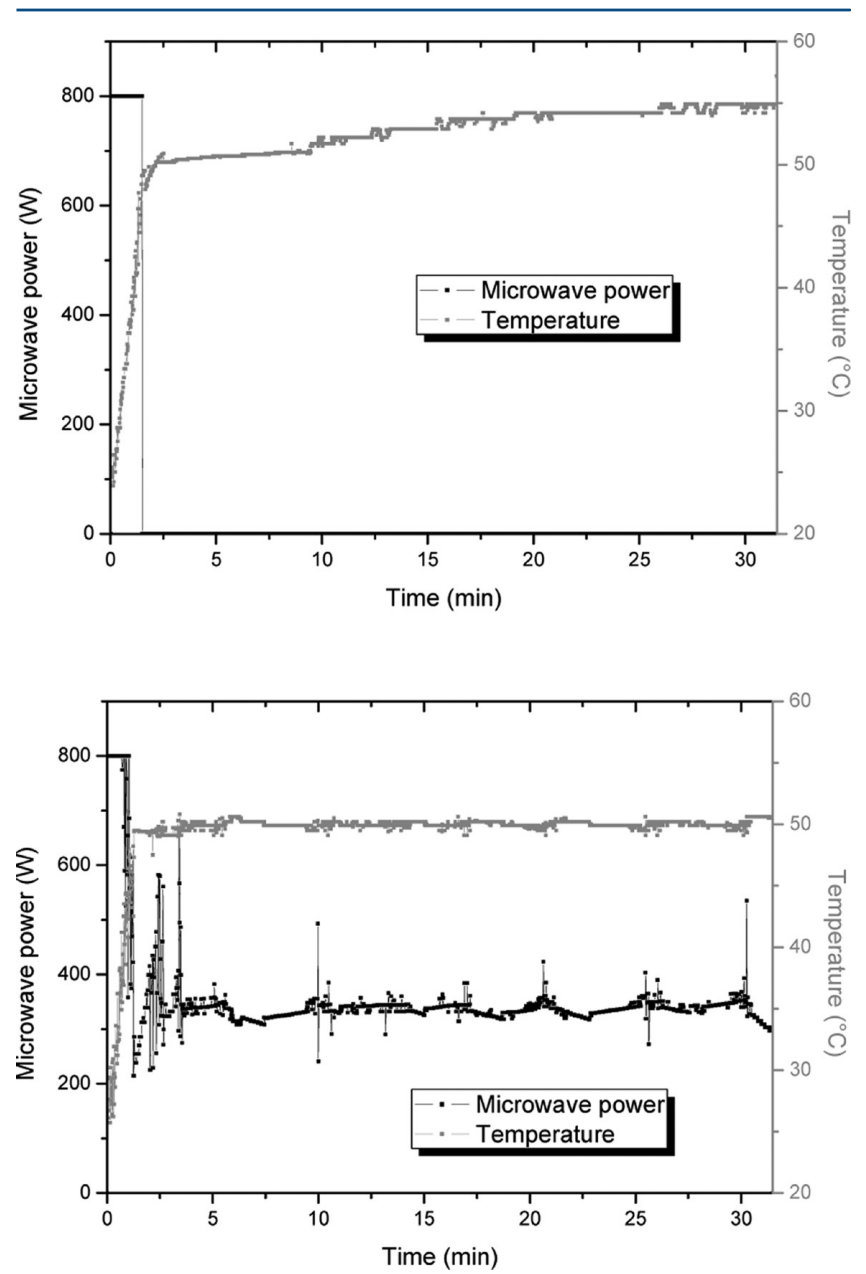

Figure 1. Typical heating curves for ultrasonic (top) pretreatments and silent pretreatments (bottom). The microwave power input is in black, and the temperature is in gray.

ultrasonic modes were chosen in order for the temperature change to have a minimal effect from increased heat from the dissipation of the ultrasonic energy in the treatment solution. Additional pretreatments in water, using conventional heating, were conducted in an oil bath. The oil bath was preheated to 50 ${ }^{\circ} \mathrm{C}$ prior to the submersion of the treatment mixture. The treatment mixture was allowed to reach $50{ }^{\circ} \mathrm{C}$, before timing of $30 \mathrm{~min}$ was commenced. The preheat time for the conventional heating pretreatments was up to $45 \mathrm{~min}$.

After an experiment, the mixture was separated by filtration through a nylon cloth with the mesh of 300 . After separation the solids were washed thoroughly (at least four times) with deionized water and dried at $39^{\circ} \mathrm{C}$ in a fan forced oven. The pretreatment liquors were refrigerated and analyzed within 2 to 3 days. Each treatment and analysis was repeated at least twice, and averages and standard deviations were reported.

2.3. Analytical Methods. 2.3.1. Solid Residue Analysis. The yield of the solid fraction was measured gravimetrically. The compositional analysis for acid insoluble lignin, cellulose, 
Table 1. Solid Residue Yields and Crystallinity and Pretreatment Liquor pHs and Free/Hydrolyzed Glucose and Xylose Ratios ${ }^{a}$

\begin{tabular}{|c|c|c|c|c|c|c|}
\hline \multirow[b]{2}{*}{ solvent } & \multirow[b]{2}{*}{ configuration } & \multicolumn{2}{|c|}{ solid analysis } & \multicolumn{3}{|c|}{ liquor analysis } \\
\hline & & solid residue yield (\%) & crystallinity index (\%) & $\mathrm{pH}$ & free/hydrolysed glucose & free/hydrolysed xylose \\
\hline \multirow[t]{3}{*}{ water } & MW & $62 \pm 4$ & $32.8 \pm 0.5$ & 5.53 & 0.8 & 1.8 \\
\hline & US & $63 \pm 2$ & $34 \pm 5$ & 5.53 & 0.8 & 2.1 \\
\hline & $\mathrm{OB}$ & $61 \pm 3$ & $36 \pm 1$ & 5.37 & 0.7 & 2.1 \\
\hline \multirow[t]{2}{*}{ PAA } & MW & $65 \pm 1$ & $34 \pm 3$ & 3.27 & 1 & 2.9 \\
\hline & US & $63 \pm 3$ & $29 \pm 4$ & 3.23 & 0.9 & 2.9 \\
\hline \multirow[t]{2}{*}{ HP } & MW & $64 \pm 2$ & $29.5 \pm 3$ & 5.15 & 2.3 & 6.7 \\
\hline & US & $63 \pm 3$ & $32.3 \pm 1$ & 5.13 & 2.5 & 7.7 \\
\hline \multirow[t]{2}{*}{ AA } & MW & $63 \pm 2$ & $29.0 \pm 0$ & 2.42 & 0.8 & 3.2 \\
\hline & US & $62 \pm 2$ & $31.1 \pm 0.4$ & 2.39 & 0.7 & 2.9 \\
\hline untreat & eat straw & & $28 \pm 3$ & & & \\
\hline
\end{tabular}

untreated wheat straw

${ }^{a}$ Water, PAA, HP, and AA denote treatment in water, peracetic acid, hydrogen peroxide, and acetic acid, respectively. US, MW, and OB denote the reactor configuration, ultrasound, microwave, and heating using an oil bath, respectively.

and hemicellulose in the solid and liquid fractions was determined following standard procedures set out by the National Renewable Energy Laboratory (NREL) ${ }^{32}$ with slight modification. All procedures outlined by NREL were followed except a Bio-Rad HPX-87H column was used with a $5 \mathrm{mmol}$ $\mathrm{L}^{-1}$ sulfuric acid eluent on a High Performance Liquid Chromatograph (HPLC) 1200 series (Agilent).

Additional analysis of the solid fractions included scanning electron microscopy (SEM) and crystallinity index determination via X-ray Diffraction (XRD) analysis. SEM images of the air-dried solid residues, coated with platinum, were captured on a Hitachi cold field SEM S-4800. XRD analysis was carried out on a D8 Advance XRD from Bruker. The crystallinity index was determined via a method outlined by Segal et al., ${ }^{33}$ using the following equation

$$
\operatorname{CrI}(\%)=100 \times \frac{\left(I_{0.02}-I_{\mathrm{am}}\right)}{I_{0.02}}
$$

where $I_{\mathrm{am}}$ represents the crystallinity for the amorphous region of the biomass at $2 \theta=18.7^{\circ}$, and $I_{0.02}$ represents the crystallinity for the crystalline portion of the biomass (cellulose) at $2 \theta=22.5^{\circ}$.

2.3.2. Pretreatment Liquor Analysis. The liquors were analyzed by measurement of the $\mathrm{pH}$, free sugar content and hydrolyzed sugar content. The free and hydrolyzed glucose and xylose concentration in the raw liquors were determined for all pretreatments following standard procedures set out by NREL. ${ }^{34}$

\section{RESULTS AND DISCUSSION}

3.1. Overall Effect of Ultrasound Compared to Silent Treatments. 3.1.1. Ultrasonic Effect on the Overall Lignocellulosic Component. No significant differences in solid residue yield were observed between ultrasound and silent pretreatments $(62-65 \%$, Table 1$)$, although differences in the composition of the solid residues were observed (Figure 2 ). For example, microwave treatments reduced the lignin content by an additional $4.6 \%, 11.9 \%, 3.1 \%$, and $2.6 \%$ for water, peracetic acid, hydrogen peroxide, and acetic acid, respectively (Figure 2). Moreover, the additional reduction of remaining hemicellulose, with microwave was $1.9 \%, 1.7 \%, 0.76 \%$, and $1.1 \%$ for water, peracetic acid, hydrogen peroxide, and acetic acid, respectively. Microwave treatment resulted in a higher reduction of cellulose content compared to ultrasound for treatment with peracetic acid and acetic acid: $2.6 \%$ and $2.9 \%$,

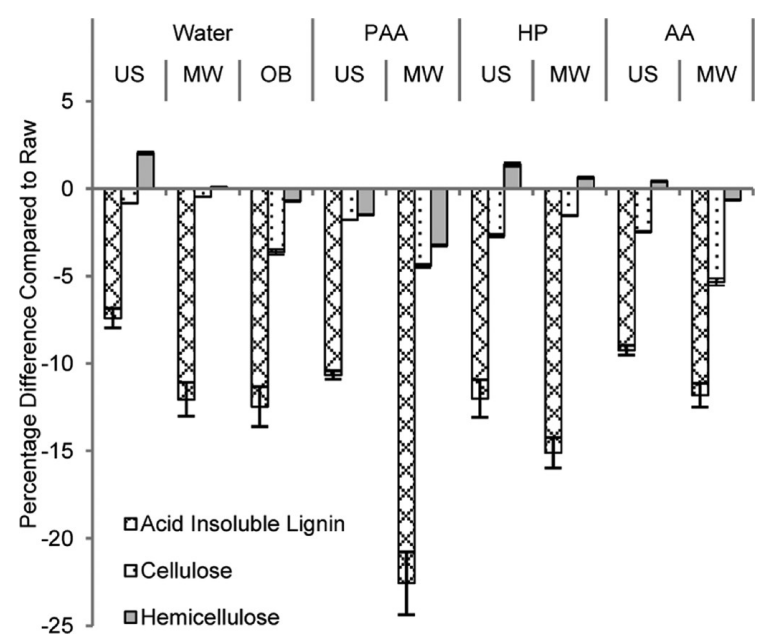

Figure 2. Changes in composition of the solid residue after different pretreatments compared to the raw material. Water, PAA, HP, and AA denote treatment in water, peracetic acid, hydrogen peroxide, and acetic acid, respectively. US, $M W$, and $O B$ denote the reactor configurations, ultrasound, microwave, and heating using an oil bath, respectively.

respectively. Conversely, for treatment with water and hydrogen peroxide, the cellulose content was reduced to a higher degree by ultrasonic treatment, by $0.37 \%$ and $1.15 \%$, respectively. In total, the composition of the biomass that corresponded to lignin, hemicellulose, and cellulose was higher for ultrasonic pretreatments than microwave pretreatments, by $1.2 \%, 3.6 \%, 0.3 \%$, and $1.8 \%$ for water, peracetic acid, hydrogen peroxide, and acetic acid, respectively. Therefore, ultrasound tended to reduce the nonlignocellulosic component of the wheat straw, more so than microwave.

The reduction of the nonlignocellulosic component was congruent with previous literature, where the contents of ash and extractives were reduced by ultrasound. ${ }^{20}$ However, the compositional analysis completed here was performed on an extractive-free basis, and hemicellulose was determined as xylose, hence the nonxylose hemicellulose was also considered. The composition of the raw wheat straw was $18.1 \%$ lignin, $37.0 \%$ glucose, and $27.3 \%$ hemicellulose (measured as xylose), which was a total of $82.3 \%$ of the dried wheat straw. This was similar to the ranges of these components found in literature: $37.7-40.2 \%$ for cellulose and $14.1-22.6 \%$ for acid insoluble lignin, but a larger variation was seen for hemicellulose. In 


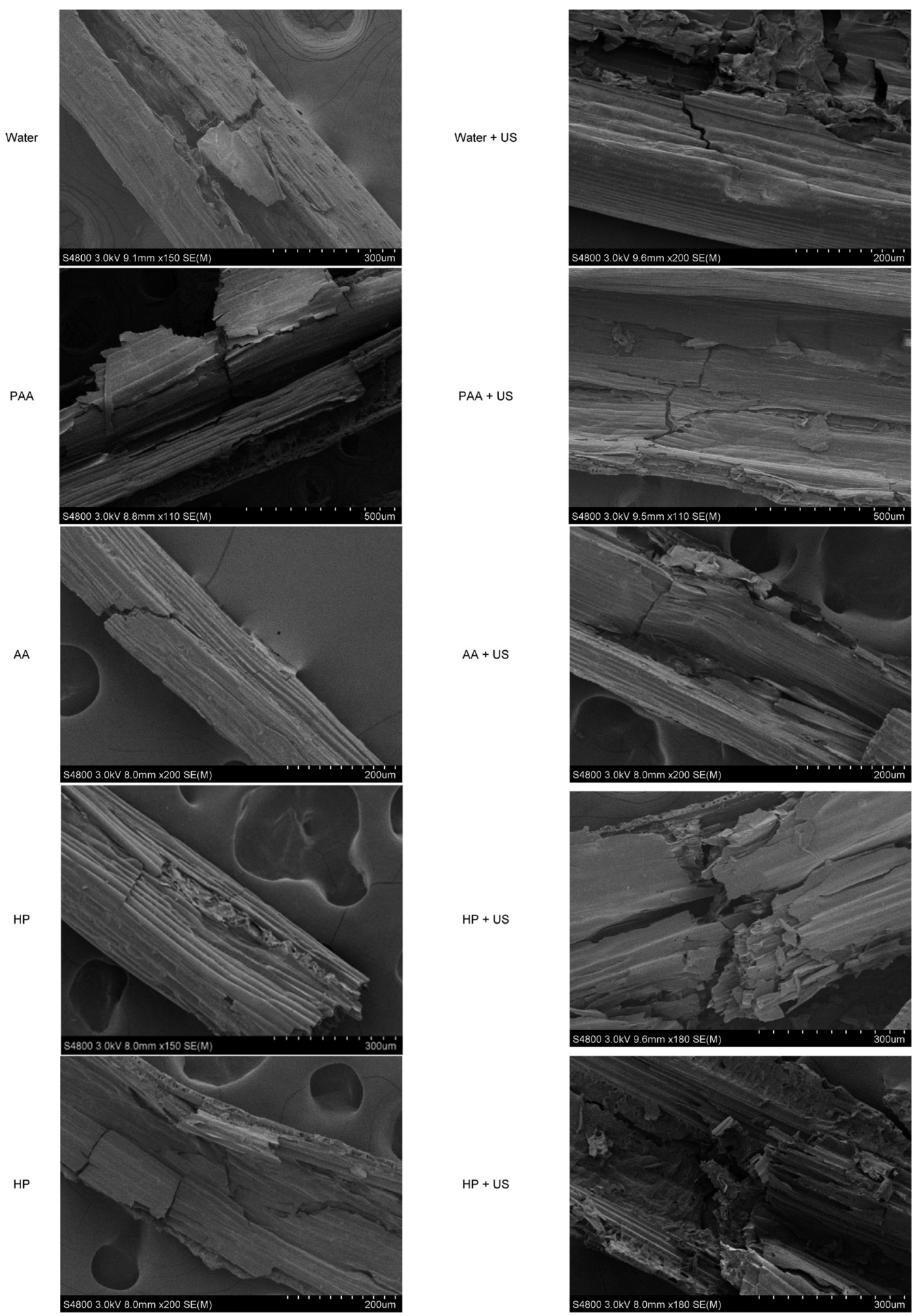

Figure 3. SEM images of pits and cracking caused by the ultrasonic pretreatments. Water, PAA, HP, and AA denote treatment in water, peracetic acid, hydrogen peroxide, and acetic acid, respectively, and US denotes ultrasound-assisted experiments.

previous reports, the hemicellulose was measured as a total of xylose, mannose, galactose, arabinose, and uronic acids and consisted of up to $39.5 \%$ of wheat straw $^{35-39}$ with xylan measured as $24.5 \%{ }^{40}$ in one instance. Thus, about $10 \%$ of the remaining composition of the wheat straw used in this study was from other hemicellulose components. Then, the additional components of pectin, inorganic residue, acid soluble lignin, and acetyl groups were likely to make up the remaining portion of the biomass. Therefore, the ultrasound enhanced the degradation of one or all of these remaining nonlignocellulosic components.

A likely contribution to the reduction of nonlignocellulosic components was from the additional hemicellulose components, not quantified in the methods of this study. A decrease in $\mathrm{pH}$ indicated an increase in acidic moieties in solution from either additional solubilization of acetyl groups from hemicellulose, or degradation of hemicellulose. ${ }^{41}$ For treatment in chemical solutions, the $\mathrm{pH}$ was lower in the liquors from 
ultrasonic treatment (Table 1), with/without ultrasound 2.39/ 2.42, 3.23/3.27, and 5.13/5.15 for acetic acid, peracetic acid, and hydrogen peroxide, respectively. Yet, the $\mathrm{pH}$ was the same for both microwave and ultrasonic heating in water (5.53). Previously, hemicellulose extracted with ultrasound had increased linearity, with a decrease in side chain structures of arabinose, glucose, galactose, and uronic acids, and was less acidic. $^{42,43}$ Moreover, when ultrasound reduced the reported ash and extractives, no trend of $\mathrm{pH}$ was observed in comparison of ultrasound to silent pretreatments. ${ }^{20}$ Therefore, in this case, the increased purity of the solid residue after ultrasonic treatment was likely to be from a reduction of additional hemicellulose components, coupled with a reduction in inorganic material.

3.1.2. Ultrasonic Effect on Delignification. Microwave-only experiments tended to reduce the lignin content compared to ultrasonic-assisted experiments. The lignin content was reduced by an additional $4.6 \%, 11.9 \%, 3.1 \%$, and $2.6 \%$ for water, peracetic acid, hydrogen peroxide, and acetic acid, respectively (Figure 2). In previous literature, microwave treatment either did not affect or reduced the lignin content, ${ }^{25,27}$ but the conditions which facilitated lignin removal with microwave were more severe $\left(190{ }^{\circ} \mathrm{C}\right)$ than the conditions used in this experiment. In addition, in this study the microwave-preheat was used for both silent and ultrasonic experiments. The highest microwave power input was during the $1.5 \mathrm{~min}$ preheat time, which was the same for both treatment settings (Figure 1). In regards to time, additional microwave time does not always add to the treatment efficacy. ${ }^{21}$ Thus, it was unlikely that the microwave mechanisms, considered at higher severities, were the reason for more effective delignification in the silent treatments in this study. Hence, lignin condensation and hemicellulose degradation were considered. Ultrasound was previously found to enable lignin condensation ${ }^{20,37,44,45}$ and sugar solubilization. ${ }^{4,46}$ However, in treatment with water, the silent treatment decreased the relative hemicellulose content compared to the ultrasonic treatment. Consequently, the lignin reduction was not due to a relative increase in hemicellulose content. Lignin condensation was observed in high biomass loadings for peracetic acid pretreatment ${ }^{11}$ and in acetic acid treatment of lignocellulose. ${ }^{47,48}$ Therefore, the combination of ultrasound with these pretreatments was likely to promote lignin condensation.

3.1.3. The Effects of Ultrasound on the Physical Characteristics of the Solid Residue. The SEM images show pits in the treated biomass, with ultrasound (Figure 3), although no differences were observed between microwave and ultrasonic treatments with respect to crystallinity (Table 1). It should be noted that the pits were only found after particle size separation to isolate the $0.5-1 \mathrm{~mm}$ particles via sieving and that no pits were observed when taking SEM images of the whole range of biomass sizes (Figure 3). The pits were often accompanied by cracks in the biomass, hence the SEM images are shown alongside cracks in the biomass which are unaccompanied by dents, observed in the nonultrasonic treatments. However, for hydrogen peroxide treatments the only possible pit was the cross-like cracking structure observed in Figure 3 (bottom two examples). The ultrasonically produced pits demonstrate the ability of ultrasonic pretreatment to increase the exposure of the biomass to the treatment solutions. The fact that the pits were only observed in the $0.5-$ $1 \mathrm{~mm}$ particle size range may present an optimal size range for ultrasonic enhancement of pretreatment for increased accessibility for wheat straw.

3.2. Ultrasonic Effects in Different Chemical Environments. 3.2.1. Peracetic Acid. Delignification. Ultrasound in combination with peracetic acid reduced delignification (Figure 2 ). With only microwave heating, peracetic acid pretreatment reduced the lignin content by $22.6 \%$. However, with ultrasound the lignin content was reduced by $10.7 \%$. Then, the hemicellulose content was also decreased more so with microwave than ultrasound, a $3.3 \%$ decrease, compared to a $1.5 \%$ decrease. Similarly, the decrease of cellulose content was higher for microwave pretreatment, a $4.4 \%$ decrease compared to $1.8 \%$. Therefore, although microwave combined with peracetic acid was more effective for delignification, this also led to an increase in sugar loss in the solid residue.

The degree of delignification with microwave heating was the highest with peracetic acid, compared to other chemical environments (Figure 2). The efficacy of peracetic acid in mild conditions over other pretreatments was also reported, compared to sulfuric acid and sodium hydroxide for delignification and enzymatic hydrolysis of sugar cane bagasse. $^{11}$ In addition, peracetic acid delignified, increased crystallinity, and increased the surface area through an exposure of fibers. ${ }^{12}$ However, under the present conditions, no significant differences in the appearance of the biomass nor in the biomass crystallinity were observed (Table 1). Although peracetic acid was successful for delignification and pretreatment of sugar cane bagasse, ${ }^{12,49}$ hybrid poplar, ${ }^{8,13}$ siam weed stem, ${ }^{10}$ and aspen biomass, ${ }^{9,14,15}$ it was not effective for pretreatment of alfala stem. ${ }^{15}$ Therefore, in this case peracetic acid was demonstrated to be viable for the pretreatment of wheat straw through improved delignification.

The delignification was nearly halved from ultrasonic treatment (Figure 2). As previously discussed in Section 3.1, lignin condensation and degradation of nonxylose components of hemicellulose was likely. However, the lignin reduction with ultrasound was halved with peracetic acid, but with all other treatments, the difference in lignin loss was between $2.6 \%$ and $4.6 \%$ for microwave and ultrasonic heating, with the lowest reduction factor being 0.62 (compared to 0.47 for peracetic acid). Therefore, the effects of ultrasound on peracetic acid were also considered.

Ultrasound has the potential to influence the mechanism of lignin degradation by peracetic acid. The mechanism of peracetic acid degradation is through hydroxonium ion attack of the electron sites within lignin, which leads to the breakdown of lignin through oxidative demethylation, ring-opening, side chain displacement, epoxidation, and cleavage of $\beta$-arylether bonds. ${ }^{3}$ After fragmentation, the acetic acid and water in the peracetic acid mixture solubilize the lignin pieces. The ultrasonic conditions, including the increased radical production and hot spots around the collapsing bubble, could enhance homolytic and thermal degradation of peracetic acid as observed in the presence of a catalyst with ultrasound ${ }^{6}$ and in a free radical system. ${ }^{50}$ Since peracetic acid delignification relies on attack from the hydroxonium ion, the homolytic degradation of peracetic acid would reduce the delignification efficacy. However, in this study, lignin degradation was still significant in the presence of ultrasound. Ultrasound has the ability to promote homolysis of peracetic acid. Thus, the delignification may have occurred via a radically driven pathway, alternate to the hydroxonium ion driven delignifica- 
tion and/or the hydroxonium ion delignification may have been reduced in ultrasonic conditions.

Carbohydrate Solubilization and Hydrolysis. During pretreatment peracetic acid released lower amounts of carbohydrates, compared to the water treatments (Figures 4

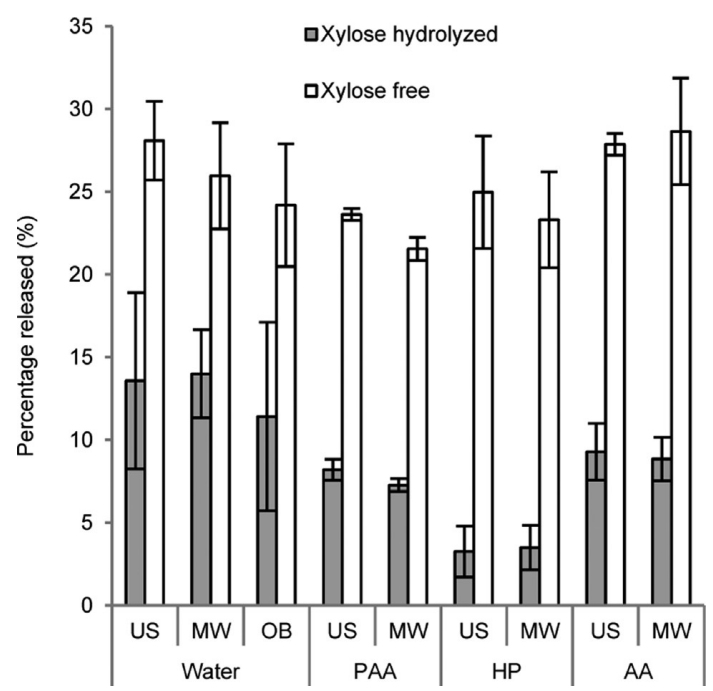

Figure 4. Xylose released in the various pretreatment liquors before and after acid hydrolysis (denoted as free and hydrolyzed xylose, respectively). Water, PAA, HP, and AA denote treatment in water, peracetic acid, hydrogen peroxide, and acetic acid, respectively. US, MW, and $\mathrm{OB}$ denote the reactor configurations, ultrasound, microwave, and heating using an oil bath, respectively.

and 5). The percentage of free xylose released with ultrasound was $23.6 \%$ compared to $28.1 \%$ for peracetic acid and water, respectively; in addition, the free xylose concentration for microwave heated pretreatment was $21.5 \%$ compared to $26.0 \%$ for peracetic acid and water, respectively. Similarly, the free glucose detected after ultrasonic pretreatment was lower for

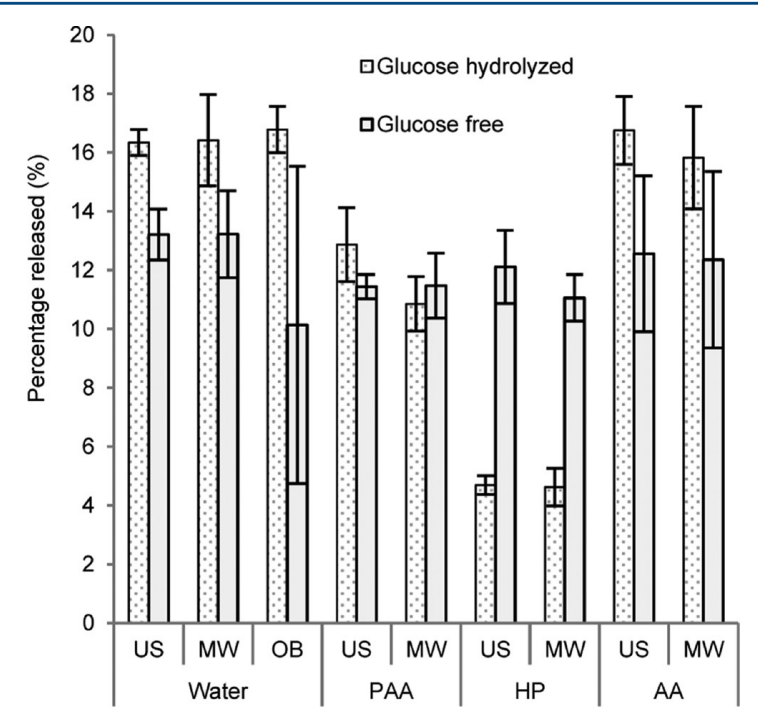

Figure 5. Glucose released in the various pretreatment liquors before and after acid hydrolysis (denoted as free and hydrolyzed glucose, respectively). Water, PAA, HP, and AA denote treatment in water, peracetic acid, hydrogen peroxide, and acetic acid, respectively. US, $\mathrm{MW}$, and $\mathrm{OB}$ denote the reactor configurations, ultrasound, microwave, and heating using an oil bath, respectively. peracetic acid pretreatment with ultrasound, compared to the water pretreatment; $11.4 \%$ compared to $13.2 \%$, despite the higher loss of hemicellulose and cellulose from the solid residue, after peracetic acid pretreatment (Figure 2). This insinuated that the peracetic acid mixture degrades glucose and xylose in solution, attributed to the presence of the sulfuric acid catalyst. $^{2,11,41}$

Free xylose released from peracetic acid microwave pretreatment was lower $(21.5 \%)$ than peracetic pretreatment with ultrasound $(23.6 \%)$, although the hydrolyzed xylose concentrations were comparable (Figure 4). This can be attributed to the depolymerization of carbohydrates in solution. ${ }^{51,52}$

The effect of the posthydrolysis conditions was examined by the comparison of the free to hydrolyzed glucose and xylose ratios (right-hand columns of Table 1). The glucose ratio from peracetic acid pretreatment was slightly higher than water (1 and 0.9 compared to 0.8 for microwave and ultrasound, respectively), and the xylose ratio was higher than water, 2.9 for both microwave and ultrasound compared to 1.8 and 2.1 for microwave and ultrasound in water. This indicated that the presence of peracetic acid has increased the xylose degradation during acid hydrolysis. This was likely to be due to the presence of hydrogen peroxide and/or acetic acid, which is discussed below.

3.2.2. Hydrogen Peroxide. Delignification. Hydrogen peroxide in ultrasonic conditions decreased the lignin content by $12 \%$ compared to $10.7 \%$ decrease for peracetic acid, $9.2 \%$ for acetic acid, and $7.4 \%$ for water. Then, with microwave heating, hydrogen peroxide pretreatment reduced the lignin content by $15.1 \%$. If the ratios of lignin loss are considered, the microwave to ultrasound loss ratio was 0.79 compared to 0.62 for hydrogen peroxide and water, respectively. This was probably because the ultrasound worked together with the hydrogen peroxide pretreatment mechanisms.

The mechanism of hydrogen peroxide delignification is via enhanced production of hydroxyl and superoxide radicals. ${ }^{18}$ In addition, an increase in temperature for pretreatment with hydrogen peroxide in alkaline conditions increased the cleavage of lignin and hemicellulose, attributed to an increase of hydroxyl radicals. ${ }^{16}$ Furthermore, the degradation of lignin was enhanced from augmentation of radical production by ultrasound in the presence of a Fenton reagent, in an ultrasonic environment. However, delignification was not improved compared to the control in the treatment of the lignocellulose. ${ }^{19}$ Also, phenol degradation with hydrogen peroxide was accelerated with ultrasound with a decrease in activation energy calculated. ${ }^{7}$ Therefore, it was proposed that the introduction of ultrasound for hydrogen peroxide treatment has enabled lignin degradation via hydroxyl and superoxide radicals.

Carbohydrate Solubilization and Hydrolysis. Without a ratio analysis, Figures 3 and 4 show a large decrease in hydrolyzed glucose and xylose. The actual free to hydrolyzed glucose ratios were 2.3 and 2.5 (compared to 0.8 for water), and the free to hydrolyzed xylose ratios were 6.7 and 7.7 (compared to 1.8 and 2.1 for water) for microwave and ultrasound, respectively (Table 1). The loss of glucose and xylose may be attributed to the increased oxidizing strength of hydrogen peroxide in acidic conditions or the exposure of sugar monomers to oxidative degradation after acid hydrolysis. ${ }^{41}$ The degradation of hemicellulose via hydrogen peroxide in acidic conditions was previously observed in solution. ${ }^{17}$ Therefore, the carbohydrates in solution were not suitable for recovery by sulfuric acid hydrolysis. 
3.2.3. Acetic Acid. For acetic acid pretreatment, the three major components of the solid residue were less affected with ultrasound. It was hypothesized that ultrasound combined with acetic acid may enhance solubilization of hemicellulose and cellulose. However, this was not observed, rather acetic acid with microwave heating produced higher loss of cellulose and hemicellulose (Figure 1). In addition, ultrasound degrades acetic acid in solution ${ }^{53}$ which would decrease the severity of the acetic acid pretreatment. Therefore, acetic acid pretreatment was not suitable for augmentation with ultrasonic conditions.

Delignification. The use of acetic acid with microwave did not improve delignification compared to water ( $11.8 \%$ loss compared to $12 \%$ loss for acetic acid and water, respectively). Then, for ultrasound there was a slightly higher loss of lignin with acetic acid (9.2\% compared to $7.4 \%$ for acetic acid and water). However, the cellulose and hemicellulose losses were both more than the losses from water pretreatment. The cellulose content was reduced by $5.3 \%$ and $2.5 \%$ for acetic acid compared to the loss in water of $0.46 \%$ and $0.84 \%$ for microwave and ultrasound, respectively. Similarly, for hemicellulose, acetic acid resulted in lower remaining percentages than water. The percentage loss for hemicellulose was $0.65 \%$ compared to a $0.09 \%$ gain for microwave pretreatment with acetic acid and water, respectively. Then, for ultrasonic pretreatment, acetic acid resulted in a gain of hemicellulose of $0.42 \%$ compared to a $2.0 \%$ gain for water. Acetic acid degrades hemicellulose and disrupts the lignin structure followed by solvation of the fragments. ${ }^{3}$ For example, $90 \%$ acetic acid was not effective for delignification in mild conditions, without an acid catalyst. ${ }^{54}$ This is congruent with the results here, which demonstrated that acetic acid was no better for delignification than water. Instead, acetic acid reduced hemicellulose and cellulose more so than water. These observations agreed with the previous literature ${ }^{20}$ where acetic acid reduced hemicellulose more so than water.

Carbohydrate Solubilization and Hydrolysis. The free and total glucose concentrations and ratios were similar to that of water (Figure 5 and Table 1). However, the free to hydrolyzed xylose ratios (Table 1) were higher than the water pretreatment. The free to hydrolyzed xylose ratios were 3.2 to 2.9 for acetic acid compared to 1.8 and 2.1 for water with microwave and ultrasonic pretreatment. This was unsurprising since sulfuric acid catalyzes hemicellulose degradation from acetic acid. $^{54}$ Thus, if glucose monomers are the desired product, acetic acid would be considered a pretreatment as an effective means of hemicellulose removal.

3.4. Thermal Heating versus Microwave Heating. The total lignocellulose composition of the solid residue, after pretreatment from thermal heating, was reduced compared to the raw biomass composition (Figure 2). In addition, thermal heating reduced the cellulose (35.6\%) and hemicellulose (27.1\%) content compared to microwave and ultrasonic treatment; $36.7 \%, 36.8 \%$ for cellulose and $27.3 \%, 27.8 \%$ for hemicellulose, respectively. Thermal heating also reduced the lignin content (15.8\%) compared to ultrasonic heating (16.7\%). The changes in lignin content compared to ultrasonic treatment support the theory that lignin condensates on the biomass under ultrasound, as previously discussed. Although both the percentage cellulose and hemicellulose in the solid residue were reduced with thermal heating pretreatment, the overall solid residue yield was not reduced. This indicated that thermal heating reduced the relative content of lignocellulose in the remaining solid residue and was congruent with the higher crystallinity observed after thermal pretreatment (36\% compared to $29 \%$ for raw) and the lower $\mathrm{pH}$ of the pretreatment liquor (5.37 compared to 5.53, Table 1). The change in crystallinity indicated that the pretreatment disrupted the hydrogen bonding in the cellulose coupled with the removal of lignin and noncellulosic polysaccharides. Then the lower $\mathrm{pH}$ shows additional support for degradation of hemicellulosic components, as previously discussed. This was likely to be due to the prolonged preheating time, of up to 45 min, to reach the desired temperature at the point of the thermometer in the middle of the reaction mixture. Microwave heating was rapid and more uniform, which did not produce areas of higher heat energy as with thermal heating which would facilitate the loss of lignocellulose components. In addition the low severity of the microwave treatment was unlikely to affect the biomass structure. Previous investigations found that microwave produced morphological changes in biomass $^{25}$ and decreased the crystallinity; ${ }^{21,27}$ however, no effects were observed here. This was attributed to the mild conditions used in this study. In previous work morphological differences were only noticeable at $130^{\circ} \mathrm{C}$ or higher, ${ }^{22}$ and loss of crystallinity was only observed at higher treatment temperatures. ${ }^{21}$ Therefore, from these experiments, microwave preheating was more efficient at obtaining the desired reaction temperature, with a higher retention of the lignocellulosic composition of the biomass, compared to thermal heating.

3.5. Outcomes of This Research. This investigation demonstrated that ultrasound increased the purity of the solid residue with respect to the main lignocellulosic components and altered the physical structure. This demonstrates that for the subsequent use of the biomass under various conditions, ultrasound is a constructive addition to pretreatment. Hence, for downstream processing of lignin, cellulose, or hemicellulose, ultrasound may augment pretreatment. However, it was also found that microwave heated pretreatments were more effective at lignin degradation. Lignin degradation is desirable for subsequent hydrolysis of hemicellulose and/or cellulose.

The potential effect of sonolysis in oxidative and nonoxidative solutions was also highlighted. It was found it was likely that hydrogen peroxide delignification was directed by ultrasonic degradation of peroxide, most likely via the production of hydroxyl and superoxide radicals. Moreover, peracetic acid was likely sonolyzed to reduce delignification via the hydroxonium ion mode and potentially delignify via alternate mechanisms. This was observed for the degradation of phenol with both hydrogen peroxide and peracetic acid with ultrasound. ${ }^{6,7}$ Similarly, the acetic acid treatment was less effective in ultrasonic conditions, likely to be from the sonolysis of acetic acid in solution. Therefore, if ultrasound is considered for pretreatment, the mode of chemical attack would benefit most if it was augmented by ultrasound.

The recoverability of carbohydrates in solution was affected by the chemical treatments. The peracetic acid and the hydrogen peroxide liquors were not suitable for recoverability via sulfuric acid hydrolysis. This would also indicate that in a biorefinery setting, recovery of the carbohydrates from the solid residue via acid hydrolysis would require thorough washing to remove peracetic acid and hydrogen peroxide. Acid hydrolysis of the acetic acid liquor only affected the hemicellulose in solution. Hence, if glucose was to be recovered, acetic acid would be a suitable pretreatment. Therefore, the use of 
chemical treatments must consider the downstream processing techniques.

The time to reach $50{ }^{\circ} \mathrm{C}$ with microwave was $1.5 \mathrm{~min}$, opposed to up to $45 \mathrm{~min}$ with thermal heating. Additionally the use of microwave resulted in a solid residue with a higher component of cellulose and hemicellulose compared to thermal heating. Previous works showed that microwave pretreatment was effective for enhanced glucose production and an increase in xylan removal. ${ }^{25,27}$ However, a comparison of microwave with ultrasonic pretreatment produced higher simultaneous saccharification and fermentation yields with microwave, ${ }^{28}$ but the saccharification and fermentation yields were from starch, not from lignocellulosic, so different results would be expected from lignocellulosic pretreatments. Therefore, microwave, as a preheat tool was more efficient and time saving.

\section{CONCLUSIONS}

Treatments with ultrasound produced solid residues with higher total percentages of lignin, xylose, and cellulose, attributed to the increased degradation of inorganic material and nonxylose components of hemicellulose. Under ultrasonic conditions, delignification was reduced, attributed to lignin condensation, and the physical structure of the biomass was altered, demonstrated by pits and cracking in the biomass.

Ultrasound combined with peracetic acid pretreatment halved the delignification efficacy and with ultrasound hydrogen peroxide was more effective at delignification than peracetic acid. This was attributed to alternate modes of interaction of ultrasound with hydrogen peroxide and peracetic acid.

\section{AUTHOR INFORMATION}

\section{Corresponding Author}

*Phone: +61 86488 8026. E-mail: Madeleine.Bussemaker@ gmail.com.

\section{Notes}

The authors declare no competing financial interest.

\section{ACKNOWLEDGMENTS}

The authors gratefully acknowledge the financial and other support provided by the Australian Research Council under the ARC Linkage Projects Scheme (ARC Linkage Project LP100200135). The authors acknowledge Mark Thomas of Treth farm for the provision of the wheat straw. Bussemaker would also like to appreciatively acknowledge the Julian Hunka scholarship provided through The University of Western Australia for provision of living expenses.

\section{REFERENCES}

(1) Kumar, P.; Barrett, D. M.; Delwiche, M. J.; Stroeve, P. Methods for pretreatment of lignocellulosic biomass for efficient hydrolysis and biofuel production. Ind. Eng. Chem. Res. 2009, 48 (8), 3713-3729.

(2) Hendriks, A. T. W. M.; Zeeman, G. Pretreatments to enhance digestibility of lignocellulosic biomass. Bioresour. Technol. 2009, 100, $10-18$.

(3) Zhao, X. B.; Cheng, K. K.; Liu, D. H. Organosolv pretreatment of lignocellulosic biomass for enzymatic hydrolysis. Appl. Microbiol. Biotechnol. 2009, 82 (5), 815-827.

(4) Bussemaker, M. J.; Zhang, D. Effect of ultrasound on lignocellulosic biomass as a pretreatment for biorefinery and biofuel applications. Ind. Eng. Chem. Res. 2013, 52 (10), 3563-3580.

(5) Mason, T. J. Applied sonochemistry: Uses of power ultrasound in chemistry and processing; Wiley VCH Verlag GmbH: 2003.

(6) Rokhina, E. V.; Makarova, K.; Lahtinen, M.; Golovina, E. A.; Van As, H.; Virkutyte, J. Ultrasound-assisted $\mathrm{MnO}_{2}$ catalyzed homolysis of peracetic6 acid for phenol degradation:The assemssment of process chemistry and kinetics. Chem. Eng. J. 2013, 221, 476-486.

(7) Rokhina, E. V.; Repo, E.; Virkutyte, J. Comparative kinetic analysis of silent and ultrasound-assisted catalytic wet peroxide oxidation of phenol. Ultrason. Sonochem. 2010, 17, 541-546.

(8) Teixeira, L. C.; Linden, J. C.; Schroeder, H. A. Alkaline and peracetic acid pretreatments of biomass for ethanol production. Appl. Biochem. Biotechnol. 1999, 77-79, 19-34.

(9) Yin, D. T.; Jing, Q.; AlDajani, W. W.; Duncan, S.; Tschirner, U. W.; Schilling, J.; Kazlauskas, R. J. Improved pretreatment of lignocellulosic biomass using enzymatically-generated peracetic acid. Bioresour. Technol. 2011, 102, 5183-5192.

(10) Zhao, X.; Zhang, L.; Liu, D. Pretreatment of Siam weed stem by several chemical methods for increasing the enzymatic digestibility. Biotechnol. J. 2010, 5, 493-504.

(11) Zhao, X. B.; Wang, L.; Liu, D. H. Effect of several factors on peracetic acid pretreatment of sugarcane bagasse for enzymatic hydrolysis. J. Chem. Technol. Biotechnol. 2007, 82 (12), 1115-1121.

(12) Zhao, X. B.; Wang, L.; Liu, D. H. Peracetic acid pretreatment of sugarcane bagasse for enzymatic hydrolysis: a continued work. J. Chem. Technol. Biotechnol. 2008, 83 (6), 950-956.

(13) Teixeira, L. C.; Linden, J. C.; Schroeder, H. A. Simultaneous saccharification and cofermentation of peracetic acid-pretreated biomass. Appl. Biochem. Biotechnol. 2000, 84-86, 111-127.

(14) Duncan, S.; Jing, Q.; Katona, A.; Kazlauskas, R. J.; Schilling, J.; Tschirner, U.; AlDajani, W. W. Increased saccharification yields from Aspen biomass upon treatment with enzymatically generated peracetic acid. Appl. Biochem. Biotechnol. 2010, 160, 1637-1652.

(15) Xu, L.; Tschirner, U. Peracetic acid pretreatment of alfalfa stem and aspen biomass. Bioresources 2012, 7 (1), 203-216.

(16) Sun, R. C.; Fang, J. M.; Tomkinson, J. Delignification of rye straw using hydrogen peroxide. Ind. Crops Prod. 2000, 12 (2), 71-83.

(17) Kim, S. B.; Um, B. H.; Park, S. C. Effect of pretreatment reagent and hydrogen peroxide on enzymatic hydrolysis of oak in percolation process. Appl. Biochem. Biotechnol. 2001, 91-93, 81-94.

(18) Gould, J. Studies on the mechanism of alkaline peroxide delignification of agricultural residues. Biotechnol. Bioeng. 1985, 27 (3), 225-231.

(19) Ninomiya, K.; Takamatsu, H.; Ohnishi, A.; Takahashi, K.; Shimizu, N. Sonocatalytic-Fenton reaction for enhanced $\mathrm{OH}$ radical generation and its application to lignin degradation. Ultrason. Sonochem. 2013, 20 (4), 1092-1097.

(20) Garcia, A.; Gonzalez Alriols, M.; Llano-Ponte, R.; Labidi, J. Ultrasound-assisted fractionation of the lignocellulosic material. Bioresour. Technol. 2011, 102, 6326-6330.

(21) Chen, W.-H.; Tu, Y.-j.; Sheen, H.-K. Disruption of sugarcane bagasse lignocellulosic structure by means of dilut sulfric acid preatreatment with microwave-assisted heating. Appl. Energy 2011, $88,2726-2734$

(22) Chen, C.; Bolder, D.; Aita, G.; Walker, M. Ethanol production from sorghum by a microwave-assisted dilute ammonia pretreatment. Bioresour. Technol. 2012, 110, 190-197.

(23) Keshwani, D. R.; Cheng, J. J. Microwave-based alkali pretreatment of switchgrass and coastal bermudagrass for bioethanol production. Biotechnol. Prog. 2009, 26 (3), 644-652.

(24) Xu, J.; Chen, H.; Kadar, Z.; Thomsen, A. B.; Schmidt, J. E.; Peng, H. Optimization of microwave pretreatment on wheat straw for ethanol production. Biomass Bioenergy 2011, 35, 3859-64.

(25) Hu, Z.; Wen, Z. Enhancing enzymatic digestibility of switchgrass by microwave-assisted alkalie pretreatment. Biochem. Eng. J. 2008, 38, 369-378.

(26) Janker-Obermeier, I.; Sieber, V.; Faulstich, M.; Schieder, D. Solubilisation of hemicellulose and lignin from wheat straw through microwave-assisted alkaline treatment. Ind. Crops Prod. 2012, 3, 198203.

(27) Pang, F.; Xue, S.; Yu, S.; Zhang, C.; Li, B.; Kang, Y. Effects of microwave power and microwave irradiation time on pretreatment efficiency and characteristics of corn stover using combination of 
steam explosion and microwave irradiation (SE-MI) pretreatment. Bioresour. Technol. 2012, 118, 111-119.

(28) Nikolic, S.; Mojovic, L.; Rakin, M.; Pejin, D.; Pejin, J. Utilization of microwave and ultrasound pretreatments in the production of bioethanol from corn. Clean Technol. Environ. Policy 2011, 13, 587594.

(29) Zhao, X. B.; Zhang, T.; Zhou, Y.; Liu, D. H. Preparation of peracetic acid from hydrogen peroxide Part 1: Kinetics for peracetic acid synthesis and hydrolysis. J. Mol. Catal. A: Chem. 2007, 271, 246252.

(30) Gong, G.; Liu, D.; Huang, Y. Microwave-assisted organic acid pretreatment for enzymatic hydrolysis of rice straw. Biosystems Eng. 2010, 107, 67-73.

(31) Koda, S.; Kimura, T.; Kondo, T.; Mitome, H. A standard method to calibrate sonochemical efficiency of an individual reaction system. Ultrason. Sonochem. 2003, 10 (3), 149-156.

(32) Sluiter, A.; Hames, B.; Ruiz, R.; Scarlata, C.; Sluiter, J.; Templeton, D.; Crocker, D. Determination of structural carbohydrates and lignin in biomass; National Renewable Energy Laboratory: Golden, 2008.

(33) Segal, L.; Creely, J. J.; Martin, A. E., Jr; Conrad, C. M. Crystallinity of native cellulose using the X-ray diffractometer. Text. Res. J. 1959, 29 (10), 786-794.

(34) Sluiter, A.; Hames, B.; Ruiz, R.; Scarlata, C.; Sluiter, A.; Templeton, D. Determination of sugars, byproducts, and degradation products in liquid fraction process samples; National Renewable Energy Laboratory: Golden, 2008.

(35) Sun, X. F.; Xu, F.; Zhao, H.; Sun, R. C.; Fowler, P.; Baird, M. S. Physicochemical characterisatio of residual hemicelluloses isolated with cyanamide-activated hydrogen peroxide from organosolv pretreated wheat straw. Bioresour. Technol. 2005, 96, 1342-1349.

(36) Sun, R. C.; Tomkinson, J.; Wang, S. Q.; Zhu, W. Characterization of lignins from wheat straw by alkaline peroxide treatment. Polym. Degrad. Stab. 2000, 67, 101-109.

(37) Sun, R.; Tomkinson, J. Comparative study of lignins isolated by alkali and ultrasound-assisted alkali extractions from wheat straw. Ultrason. Sonochem. 2002, 9 (2), 85-93.

(38) Sun, X. F.; Sun, R. C.; Fowler, P.; Baird, M. S. Extraction and characterization of original lignin and hemicelluloses from wheat straw. J. Agric. Food Chem. 2005, 53, 860-870.

(39) Singh, D.; Zeng, J.; Laskar, D. D.; Deobald, L.; Hiscox, W. C.; Chen, $S$. Investigation of wheat straw biodegradation by Phanerochaete chrysospium. Biomass Bioenergy 2011, 35, 1030-1040.

(40) Kristensen, J. B.; Thygesen, L. G.; Felby, C.; Jorgensen, H.; Elder, T., Cell-wall structural chaninges in wheat straw pretreated for bioethanol production. Biotechnol. Biofuels 2008, 1, (5).

(41) Hon, D. N.-S.; Shiraishi, N. Wood and Cellulosic Chemistry; Marcel Dekker: Basel, 2001.

(42) Sun, R; Tomkinson, J. Characterization of hemicelluloses obtained by classical and ultrasonically assisted extractions from wheat straw. Carbohydr. Polym. 2002, 50, 263-271.

(43) Sun, R. C.; Sun, X. F.; Ma, X. H. Effect of ultrasound on the structural and physicochemical properties of organosolve soluble hemicelluloses from wheat straw. Ultrason. Sonochem. 2002, 9, 95101.

(44) Sul'man, E. M.; Sul'man, M. G.; Prutenskaya, E. A. Effect of ultrasonic pretreatment on the composition of lignocellulosic material in biotechnologiacal processes. Biocatalysis 2011, 3 (1), 28-33.

(45) Garcia, A.; Gonzalez Alriols, M.; Labidi, J. Evaluation of the effect of ultrasound on organosolv black liquor from olive tree pruning residues. Bioresour. Technol. 2012, 108, 155-161.

(46) Ebringerova, A.; Hromadkova, Z. An overview on the application of ultrasound in extraction, separation and purification of plant polysaccharides. Cent. Eur. J. Chem. 2010, 8 (2), 243-257.

(47) Vazquez, G.; Antorrena, G.; Gonzalez, J.; Freire, S.; Crespo, I. The influence of acetosolv pulping conditions on the enzymatic hydrolysis of Eucalyptus pulps. Wood Sci. Technol. 2000, 34, 345-354.

(48) McDonough, T. J. The chemistry of organosolv delignification. Tappi J. 1993, 76 (8), 186-193.
(49) Zhao, X.; Peng, F.; Cheng, K.; Liu, D. Enhancement of the enzymatic digestibility of sugarcane bagasse by alkali-peracetic acid pretreatment. Enzyme Microb. Technol. 2009, 44 (1), 17-23.

(50) Rokhina, E. V.; Makarova, K.; Golovina, E. A.; Van As, H.; Virkutyte, J. Free radical reaction pathway, thermochemistry of peracetic acid homolysis, and its application for phenol degradation: Spectroscopic study and quantum chemistry calculations. Environ. Sci. Technol. 2010, 44, 6815-6821.

(51) Ebringerova, A.; Hromadkova, Z. The effect of ultrasound on the structure and properties of the water-soluble corn hull heteroxylan. Ultrason. Sonochem. 1997, 4 (4), 305-309.

(52) Kardos, N.; Luche, J. L. Sonochemistry of carbohydrate compounds. Carbohydr. Res. 2001, 332 (2), 115-131.

(53) Findik, S.; Gunduz, G.; Gunduz, E. Direct sonication of acetic acid in aqueous solutions. Ultrason. Sonochem 2006, 13 (3), 203-207.

(54) Li, M.-F.; Sun, S.-N.; Xu, F.; Sun, R.-C. Mild acetosolv process to fractionate bamboo for the biorefinery: Structural and antioxidant properties of the dissolved lignin. J. Agric. Food Chem. 2012, 60, 17031712. 